\title{
A Multiagent System Manages Collaboration in Emergent Processes
}

\author{
John Debenham \\ Faculty of Information Technology \\ University of Technology, Sydney \\ NSW, Australia \\ debenham@it.uts.edu.au
}

\begin{abstract}
Emergent processes are non-routine, collaborative business processes whose execution is guided by the knowledge that emerges during a process instance. In so far as the process goal gives direction to conventional business processes, the continually evolving process knowledge gives direction to emergent processes. Emergent processes may involve informal interaction, and so there is a limit to the extent to which the processes can be "managed". The collaboration however can be managed. Managing collaboration needs an intelligent agent that is guided not by a process goal, but by observing the performance of the other agents. Each agent has process knowledge - that is information either generated by the individual users or is extracted from the environment, and performance knowledge - that describes how the other agents, together with their 'owners', perform - including how reliable they are. The integrity of the information derived from past observations decays in time, and so they have an inference mechanism that can cope with information of decaying integrity. An agent is described that achieves this by using ideas from information theory. The agents' internal representation language is probabilistic first-order logic. They derive models of the other agents using entropy-based inference that is based on random worlds. Maximum entropy inference is used to construct these models that are then refreshed as new information is received using minimum relative entropy inference.
\end{abstract}

\section{Categories and Subject Descriptors}

I.2.11 [Artificial Intelligence]: Distributed Artificial Intelligence-Multiagent systems

\section{General Terms}

Theory

Permission to make digital or hard copies of all or part of this work for personal or classroom use is granted without fee provided that copies are not made or distributed for profit or commercial advantage and that copies bear this notice and the full citation on the first page. To copy otherwise, to republish, to post on servers or to redistribute to lists, requires prior specific permission and/or a fee.

AAMAS'05, July 25-29, 2005, Utrecht, Netherlands.

Copyright 2005 ACM 1-59593-094-9/05/0007 ...\$5.00.

\section{Keywords}

Business process management, negotiation, collaboration

\section{INTRODUCTION}

Emergent processes are business processes that are not predefined and are ad hoc. These processes typically take place at the higher levels of organisations [9], and are distinct from production workflows [5]. Emergent processes are opportunistic in nature whereas production workflows are routine [3]. How an emergent process will terminate may not be known until the process is well advanced. The tasks involved in an emergent process are typically not predefined and emerge as the process develops. Those tasks may be carried out by collaborative groups as well as by individuals [15] and may involve informal meetings, business lunches and so on. Further, the goal of an emergent process instance may mutate as the instance matures. So unlike "lower-order" processes, the goal of an emergent process instance may not be used as a focus for the process management system. An emergent process may have a fixed goal such as "maximise profits" - but it is unlikely that a process management agent, or a human agent, will have an executable plan to achieve such a goal. Emergent processes may involve informal interaction, and so there is a limit to the extent to which the processes can be "managed". The collaboration however can be managed, and that is addressed here.

The term "business process management" [14] is generally used to refer to the simpler class of workflow processes [5], although there are notable exceptions [11]. From the management perspective, emergent processes are "knowledgedriven". A knowledge-driven process is guided by its "process knowledge" and "performance knowledge". Process knowledge is information either generated by the individual users or is extracted from the environment, and includes background information. Performance knowledge describes how the other agents together with their 'owners' perform, including how reliable they are.

In so far as the process goal gives direction to goal-driven processes, the continually evolving process knowledge gives direction to knowledge-driven processes. So plan-based agent architectures such as BDI [17] are not directly suitable. To manage knowledge-driven process with such an architecture would require machinery to manage the mutations of the process goal. The agent architecture described here manages the collaboration in the processes, and not the processes themselves. This architecture is based on ideas from information theory. 


\section{PROCESS MANAGEMENT}

Following [5] a business process is "a set of one or more linked procedures or activities which collectively realise a business objective or policy goal, normally within the context of an organisational structure defining functional roles and relationships". Implicit in this definition is the idea that a process may be repeatedly decomposed into linked sub-processes until those sub-processes are activities which are atomic pieces of work. [viz [5] "An activity is a description of a piece of work that forms one logical step within a process."].

A particular process is called a (process) instance. An instance may require that certain things should be done; such things are called tasks. Three classes of business process are defined in terms of their management properties.

- A task-driven process has a unique decomposition into a - possibly conditional - sequence of activities. Each of these activities has a goal and is associated with a task that "always" achieves this goal. These process are often called production workflow. They may be managed by reactive agents - and do not necessarily require deliberative agents or intricate management machinery.

- A goal-driven process has a process goal, and achievement of that goal is the termination condition for the process. The process goal may have various decompositions into sequences of sub-goals where these subgoals are associated with (atomic) activities and so with tasks. Some of these sequences of tasks may work better than others, and there may be no way of knowing which is which [15]. The possibility of task failure is a feature of goal-driven processes. In any case, a goal-driven process management system requires a mechanism for selecting plans for sub-goals. A simplified view of goal-driven process management is shown in Fig. 1. In that figure "SC" refers to the success condition that is a procedure at the end of each path through a plan that determines whether or not the plan has achieved its goal. Activities are atomic subprocesses.

- A knowledge-driven process may have a process goal, but the goal may be at a high-level, or may be vague and may mutate [3] leading to a revised process goal. Mutations are determined by the knowledge generated during the process. At each stage in the performance of a knowledge-driven process, the "next goal" is identified using the process knowledge. So in so far as the process goal gives direction to goal-driven - and taskdriven - processes, the process knowledge gives direction to knowledge-driven processes. A simplified view of knowledge-driven process management is shown in Fig. 2.

The complete representation, never mind the maintenance, of the process knowledge may be complex. However, in a process management system, or in an Electronic Institution [4], much of that knowledge may be readily available. Performance knowledge is not difficult to capture, represent and maintain. For example, measurements of how long another agent took to complete a sub-process, and measurements of how reliable the other agents are.
Figure 1: Goal-driven processes.

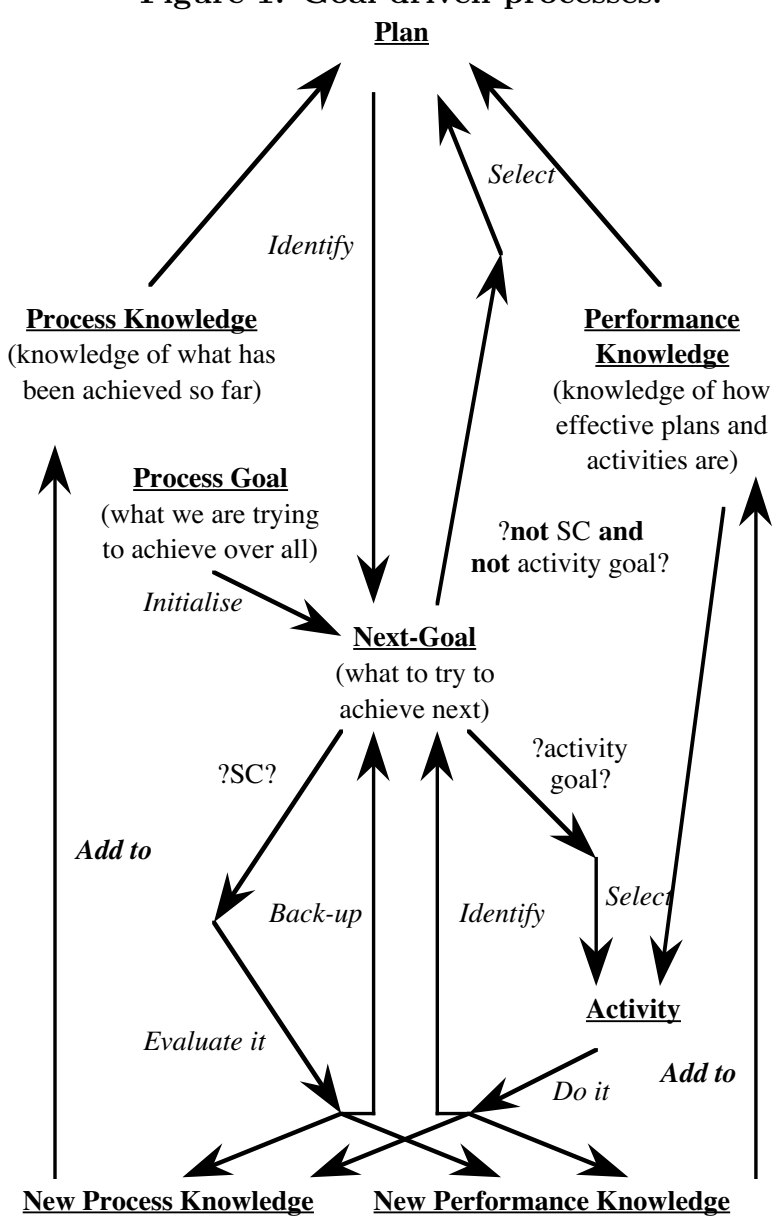

\section{EMERGENT PROCESS MANAGEMENT SYSTEM}

In the system described here each human player is assisted by an agent. As emergent processes may involve informal interaction between players, there is a limit to the extent to which the processes themselves can be "managed". This is in contrast to task-driven processes, or production workflow, where a management system prescribes what should happen next - agents are "asked" to do things and are "expected" to comply. For emergent process the collaboration can be managed. The questions that an emergent process agent considers include: "who to ask to assist", "who can I rely on", "who works well with who", "who do I want to build a relationship with". The answers to these questions are inferred by observing the dynamics of the collaboration between the agents. So an agent for emergent process management needs to be able to observe and evaluate the collaboration - what appears to work and what does not and has to make sense out of this diverse information.

The act of an agent joining a real or virtual group for some purpose is fundamental to collaboration. Another act is one agent delegating responsibility for a sub-process to another. The product of group activity, or process delegation, is some information being generated, and so to the act of information being passed from one agent to another. The system 
Figure 2: Knowledge-driven processes.

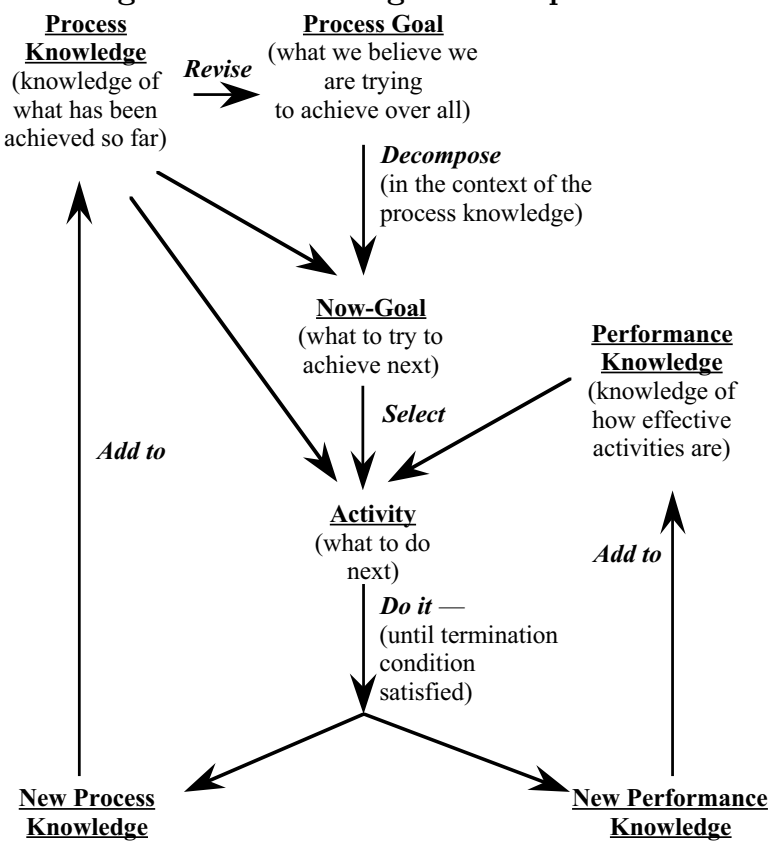

aims to implement these three types of act intelligently. It consists of the set of agents $\left\{X_{i}\right\}_{i=0}^{n}$ - the description following is written from the point of view of agent $X_{0}$ that interacts with the other $n$ agents. In the text, the agent $X_{\omega}$ is "an other" agent - i.e. $\omega \neq 0$.

The agent architecture extends the agent described in [2]. It is driven by the contents of a knowledge base that represents the agent's world model represented in probabilistic first-order logic. The system attempts to manage the collaboration using the information that is generated both by and because of it. To achieve this, it draws on ideas from information theory. As with the agent described in [2], $X_{0}$ makes assumptions about: the way in which the integrity of information will decay, and some of the preferences that its collaborators may have for some agreements over others. It also assumes that unknown probabilities can be inferred using maximum entropy inference [12], $M E$, which is based on random worlds [7]. The maximum entropy probability distribution is "the least biased estimate possible on the given information; i.e. it is maximally noncommittal with regard to missing information" [10]. In the absence of knowledge about the other agents' allegiances, $X_{0}$ assumes that the "maximally noncommittal" model is the correct model on which to base its reasoning.

$X_{0}$ decides what to do - such as what message to send - on the basis of its past observations, the current integrity of which is expressed as degrees of belief. $X_{0}$ uses this information to calculate, and continually revise, probability distributions for that which it does not know. One such distribution, over the set of all possible actions, expresses $X_{0}$ 's belief in the suitability to herself of performing that action. Other distributions attempt to predict the behaviour of another agent — such as what agreements she might accept. $X_{0}$ is purely concerned with the other agents' behaviours what they actually do - and not with assumptions about their motivations. This somewhat detached stance is ap- propriate for emergent process management in which each agent represents the interests of it owner, whilst at the same time attempting to achieve social goals.

\section{EMERGENT PROCESS AGENT $x_{0}$}

$X_{0}$ operates in an information-rich environment that includes the Internet. One source of $X_{0}$ 's information is the signals received from $X_{\omega}$. These include proposals from $X_{\omega}$ to $X_{0}$, the acceptance or rejection by $X_{\omega}$ of $X_{0}$ 's proposals, and information that $X_{\omega}$ sends to $X_{0}$. Incoming information is augmented by $X_{0}$ with sentence probabilities that represent the strength of her belief in its truth. If $X_{\omega}$ refused to assist $X_{0}$ two days ago then what is $X_{0}$ 's belief now in the proposition that $X_{\omega}$ will assist her now? Perhaps it is around 0.1 . For simplicity, a linear model is used to model the integrity decay of these beliefs, and when the probability of a decaying belief approaches its maximum entropy value the belief is discarded.

\subsection{Interaction Protocol}

An agreement is a pair of commitments $\delta_{X_{0}: X_{\omega}}\left(x_{0}, x_{\omega}\right)$ between an agent $X_{0}$ and another agent $X_{\omega}$, where $x_{0}$ is $X_{0}$ 's commitment and $x_{\omega}$ is $X_{\omega}$ 's commitment. $\mathcal{A}=\left\{\delta_{i}\right\}_{i=1}^{D}$ is the agreement set - ie: the set of all possible agreements. If the context is clear then the subscript " $X_{0}: X_{\omega}$ " is omitted. These commitments may involve multiple issues - not simply a single issue such as time to complete a task. The set of terms, $\mathcal{T}$, is the set of all possible commitments that could occur in an agreement $a \in \mathcal{A}$.

An agent may have a real-valued utility function: $\mathbf{U}: \mathcal{T} \rightarrow$ $\Re$, that induces an ordering on $\mathcal{T}$. For such an agent, for any agreement $\delta=\left(x_{0}, x_{\omega}\right)$ the expression $\mathbf{U}\left(x_{\omega}\right)-\mathbf{U}\left(x_{0}\right)$ is called the surplus of $\delta$, and is denoted by $\mathbf{L}(\delta)$ where $\mathbf{L}: \mathcal{T} \times \mathcal{T} \rightarrow \Re$. For example, the values of the function $\mathbf{U}$ may expressed in units of time. It may not be possible to specify the utility function either precisely or with certainty. This is addressed in Sec. 5 .

The agents communicate in an illocutionary language, $\mathcal{C}$, that has the illocution particle set:

$$
\iota=\{\text { Delegate, Join, Accept, Reject, Inform, Quit }\}
$$

with the following syntax and informal meaning:

- Delegate $\left(\left(X_{0}, \rho\right),\left(X_{\omega}, G_{i}\right)\right)$ means " $X_{0}$ proposes to recompense $X_{\omega}$ with $\rho$ if $X_{\omega}$ agrees to take responsibility for an individual goal $G_{i} "$.

- Join $\left(\left(X_{0}, \rho\right),\left(X_{\omega}, G_{i}\right)\right)$ means " $X_{0}$ proposes to recompense $X_{\omega}$ with $\rho$ if $X_{\omega}$ agrees to contribute to cooperative goal $G_{j}$ ".

- Accept $(\delta)$ means "the sender accepts your proposed agreement $\delta "$.

- Reject $(\delta)$ means "the sender rejects your proposed agreement $\delta$.

- $\operatorname{Inform}\left(\left(X_{0}, \mathcal{I}_{k}\right), X_{\omega}\right)$ means " $X_{0}$ offers information $\mathcal{I}_{k}$ to $X_{\omega} "$.

- Quit (.) means "the sender quits - the interaction ends".

So for these predicates, and in this discussion, an agreement $\delta$ has the form $\left(\left(X_{0}, \rho\right),\left(X_{\omega}, G_{i}\right)\right)$. 
The communication predicates described in the previous paragraph introduce a number of concepts. In the interest of brevity these are only described here informally. The notion of one agent recompensing another [i.e. $\rho$ ] refers to both the informal "thanks, I owe you one", and to the formal "take the rest of the day off", or some sum of money. An individual goal has the form of: information $\mathcal{I}_{k}$ will be sent to agent $X_{r}$ by time $t$. An cooperative goal has the form of: the assembly of information $\mathcal{I}_{k}$ will be co-ordinated by agent $X_{0}$ by time $t$. The expression of the information requires some ontology - that is not described here.

\subsection{Agent Architecture}

$X_{0}$ uses the language $\mathcal{C}$ for external communication, and the language $\mathcal{L}$ for internal representation. One predicate in $\mathcal{L}$ is: $\operatorname{Acc}_{\mathrm{d}}\left(\left(\left(X_{0}, \rho\right),\left(X_{\omega}, G_{i}\right)\right)\right)$. The proposition $\left(\operatorname{Acc}_{\mathrm{d}}(\delta) \mid \mathcal{I}_{t}\right)$ means: " $X_{0}$ will be comfortable accepting the delegation agreement $\delta$ with agent $X_{\omega}$ given that $X_{0}$ knows information $\mathcal{I}_{t}$ at time $t$ ". The idea is that $X_{0}$ will accept delegation agreement $\delta$ if $\mathbf{P}\left(\operatorname{Acc}_{\mathrm{d}}(\delta) \mid \mathcal{I}_{t}\right) \geq \alpha$ for some threshold constant $\alpha$. The precise meaning that $X_{0}$ gives to $\operatorname{Acc}_{\mathrm{d}}(\delta)$ is described in Sec. 5. Similarly $\operatorname{Acc}_{j}$ for Join $(\cdot)$ agreements. The probability distribution $\mathbf{P}\left(\operatorname{Agg}_{\mathrm{d}}\left(\left(X_{0}, \rho\right),\left(X_{\omega}, G_{i}\right)\right)\right)$ is agent $X_{0}$ 's estimate of the probability that agent $X_{\omega}$ will agree to the Delegate agreement $\delta$ [or $\operatorname{Agg}_{\mathrm{j}}(\cdot)$ for Join( $(\cdot)$ agreements] - it is estimated in Sec. 6.

Each incoming message $M$ from source $S$ received at time $t$ is time-stamped and source-stamped, $M_{[S, t]}$, and placed in an in box, $\mathcal{X}$, as it arrives. $X_{0}$ has an information repository $\mathcal{I}$, a knowledge base $\mathcal{K}$ and a belief set $\mathcal{B}$. Each of these three sets contains statements in a first-order language $\mathcal{L}$. $\mathcal{I}$ contains statements in $\mathcal{L}$ together with sentence probability functions of time. $\mathcal{I}_{t}$ is the state of $\mathcal{I}$ at time $t$ and may be inconsistent. At some particular time $t, \mathcal{K}_{t}$ contains statements that $X_{0}$ believes are true at time $t$, such as $\forall x(\operatorname{Accept}(x) \leftrightarrow \neg \operatorname{Reject}(x))$. The belief set $\mathcal{B}_{t}=\left\{\beta_{i}\right\}$ contains statements that are each qualified with a given sentence probability, $\mathbf{B}\left(\beta_{i}\right)$, that represents $X_{0}$ 's belief in the truth of the statement at time $t$. The distinction between the knowledge base $\mathcal{K}$ and the belief set $\mathcal{B}$ is simply that $\mathcal{K}$ contains unqualified statements and $\mathcal{B}$ contains statements that are qualified with sentence probabilities. $\mathcal{K}$ and $\mathcal{B}$ play different roles in the method described in Sec. 4.3; $\mathcal{K}_{t} \cup \mathcal{B}_{t}$ is required by that method to be consistent.

$X_{0}$ 's actions are determined by its "strategy". A strategy is a function $\mathbf{S}: \mathcal{K} \times \mathcal{B} \rightarrow \mathcal{A}$ where $\mathcal{A}$ is the set of actions. At certain distinct times the function $\mathbf{S}$ is applied to $\mathcal{K}$ and $\mathcal{B}$ and the agent does something. The set of actions, $\mathcal{A}$, includes sending Delegate $(\cdot)$, Join $(\cdot)$, Accept $(\cdot)$, Reject $(\cdot)$, $\operatorname{Inform}(\cdot)$ and Quit $(\cdot)$ messages to $X_{\omega}$. The way in which $\mathbf{S}$ works is described in Secs. 6. Two "instants of time" before the $\mathbf{S}$ function is activated, an "import function" and a "revision function" are activated. The import function I : $\left(\mathcal{X} \times \mathcal{I}_{t^{-}}\right) \rightarrow \mathcal{I}_{t}$ clears the in-box, using its "import rules". An import rule takes a message $M$, written in language $\mathcal{C}$, and from it derives sentences written in language $\mathcal{L}$ to which it attaches decay functions, and adds these sentences together with their decay functions to $\mathcal{I}_{t}-$ to form $\mathcal{I}_{t}$. These decay functions are functions of the message type, the time the message arrived and the source from which it came - an illustration is given below. An import rule has the form: $\mathbf{P}\left(S \mid M_{\left[X_{\omega}, t\right]}\right)=f\left(M, X_{\omega}, t\right) \in[0,1]$, where $S$ is a statement, $M$ is a message and $f$ is the decay function. Then the belief revision function $\mathbf{R}: \mathcal{I}_{t^{-}} \rightarrow\left(\mathcal{I}_{t} \times \mathcal{K}_{t} \times \mathcal{B}_{t}\right)$ deletes any statements in $\mathcal{I}_{t^{-}}$whose sentence probability functions have a value that is $\approx 0.5$ at time $t$. From the remaining statements $\mathbf{R}$ selects a consistent set of statements, instantiates their sentence probability functions to time $t$, and places the unqualified statements from that set in $\mathcal{K}_{t}$ - the qualified statements, together with their sentence probabilities, are placed in $\mathcal{B}_{t}$

$X_{0}$ uses three things to construct proposals: an estimate of the likelihood that $X_{\omega}$ will accept any agreement [Sec. 6], an estimate of the likelihood that $X_{0}$ will, in hindsight, feel comfortable accepting any particular agreement [Sec. 5], and an estimate of when $X_{\omega}$ may quit and leave the interaction - see $[2]$.

\subsection{Inference}

$X_{0}$ employs maximum entropy inference and minimum relative entropy inference to derive expectations of future performance from prior, sparse observations. Let $\mathcal{G}$ be the set of all positive ground literals that can be constructed using the symbols in $\mathcal{L}$. A possible world is a valuation function $\mathcal{V}: \mathcal{G} \rightarrow\{\top, \perp\}$. $\mathcal{V}$ denotes the set of all possible worlds, and $\mathcal{V}_{\mathcal{K}}$ denotes the set of possible worlds that are consistent with a knowledge base $\mathcal{K}[7]$.

A random world for $\mathcal{K}$ is a probability distribution $\mathcal{W}_{\mathcal{K}}=$ $\left(p_{i}\right)$ over $\mathcal{V}_{\mathcal{K}}=\left(\mathcal{V}_{i}\right)$, where $\mathcal{W}_{\mathcal{K}}$ expresses an agent's degree of belief that each of the possible worlds is the actual world. The derived sentence probability of any $\sigma \in \mathcal{L}$, with respect to a random world $\mathcal{W}_{\mathcal{K}}$ is $(\forall \sigma \in \mathcal{L})$ :

$$
\mathbf{P}_{\mathcal{W}_{\mathcal{K}}}(\sigma) \triangleq \sum_{n}\left\{p_{n}: \sigma i s \top \text { in } \mathcal{V}_{n}\right\}
$$

A random world $\mathcal{W}_{\mathcal{K}}$ is consistent with the agent's beliefs $\mathcal{B}$ if: $(\forall \beta \in \mathcal{B})\left(\mathbf{B}(\beta)=\mathbf{P}_{\mathcal{W}_{\mathcal{K}}}(\beta)\right)$. That is, for each belief its derived sentence probability as calculated using Eqn. 1 is equal to its given sentence probability.

The entropy of a discrete random variable $X$ with probability mass function $\left\{p_{i}\right\}$ is [12]: $\mathbf{H}(X)=-\sum_{n} p_{n} \log p_{n}$ where: $p_{n} \geq 0$ and $\sum_{n} p_{n}=1$. Let $\mathcal{W}_{\{\mathcal{K}, \mathcal{B}\}}$ be the "maximum entropy probability distribution over $\mathcal{V}_{\mathcal{K}}$ that is consistent with $\mathcal{B}$ ". Given an agent with $\mathcal{K}$ and $\mathcal{B}$, maximum entropy inference states that its derived sentence probability for any sentence, $\sigma \in \mathcal{L}$, is:

$$
(\forall \sigma \in \mathcal{L}) \mathbf{P}(\sigma) \triangleq \mathbf{P}_{\mathcal{W}_{\{\mathcal{K}, \mathcal{B}\}}}(\sigma)
$$

Using Eqn. 2, the derived sentence probability for any belief, $\beta_{i}$, is equal to its given sentence probability. So the term sentence probability is used without ambiguity.

If $X$ is a discrete random variable taking a finite number of possible values $\left\{x_{i}\right\}$ with probabilities $\left\{p_{i}\right\}$ then the entropy is the average uncertainty removed by discovering the true value of $X$, and is given by $\mathbf{H}(X)=-\sum_{n} p_{n} \log p_{n}$. The maximum entropy distribution: $\arg \max _{\underline{p}} \mathbf{H}(\underline{p}), \underline{p}=$ $\left(p_{1}, \ldots, p_{N}\right)$, subject to $M+1$ linear constraints:

$$
\begin{aligned}
& g_{j}(\underline{p})=\sum_{i=1}^{N} c_{j i} p_{i}-\mathbf{B}\left(\beta_{j}\right)=0, \quad j=1, \ldots, M . \\
& g_{0}(\underline{p})=\sum_{i=1}^{N} p_{i}-1=0
\end{aligned}
$$

where $c_{j i}=1$ if $\beta_{j}$ is $\top$ in $v_{i}$ and 0 otherwise, and $p_{i} \geq$ $0, i=1, \ldots, N$, is found by introducing Lagrange multipli- 
ers, and then obtaining a numerical solution using the multivariate Newton-Raphson method. In the subsequent subsections we'll see how an agent updates the sentence probabilities depending on the type of information used in the update.

Given a prior probability distribution $q=\left(q_{i}\right)_{i=1}^{n}$ and a set of constraints, the principle of minimum relative entropy chooses the posterior probability distribution $p=\left(p_{i}\right)_{i=1}^{n}$ that has the least relative entropy with respect to $q$ :

$$
\arg \min _{\underline{p}} \sum_{i=1}^{n} p_{i} \log \frac{p_{i}}{q_{i}}
$$

and that satisfies the constraints. The principle of minimum relative entropy is a generalisation of the principle of maximum entropy. If the prior distribution $q$ is uniform, the relative entropy of $p$ with respect to $q$ differs from $-\mathbf{H}(p)$ only by a constant. So the principle of maximum entropy is equivalent to the principle of minimum relative entropy with a uniform prior distribution.

\section{ACCEPTABILITY OF A PROPOSAL.}

Why would $X_{0}$ accept a Delegate $(\cdot)$ or a Join $(\cdot)$ proposal? Each deal, $\delta=\left(\left(X_{0}, \rho\right),\left(X_{\omega}, G_{i}\right)\right)$, contains provision for an incentive $\rho$. However it is more realistic [16] to assume that the agents in an emergent process management system are benevolent [8] - that is, they will accept a responsibility for a process if they believe that they can achieve the process goal. So $X_{0}$ needs machinery to estimate the probability that if it takes responsibility for goal $G_{i}$ then it will achieve it. Sec. 6 considers the converse problem: that is, how $X_{0}$ estimates the probability distribution over all possible responses that $X_{\omega}$ will respond in various ways.

The proposition $\left(\operatorname{Acc}_{\mathrm{d}}\left(\left(X_{0}, \rho\right),\left(X_{\omega}, G_{i}\right)\right) \mid \mathcal{I}_{t}\right)$ was introduced in Sec. 4.2. This section describes how the agent estimates $\mathbf{P}\left(\operatorname{Acc}_{\mathrm{d}}(\delta) \mid \mathcal{I}_{t}\right)$ - i.e. the probability that $X_{0}$ attaches to the truth of this proposition for various $\delta$. This is described for delegations only - Join $(\cdot)$ is dealt with similarly.

$X_{0}$ forms its future expectations on the basis of past observations, including the expectations that it has about itself. Sec. 6 following describes how $X_{0}$ forms its expectations about a collaborator. The same approach is used estimate $\mathbf{P}\left(\operatorname{Acc}_{\mathrm{d}}\left(\left(X_{0}, \rho\right),\left(X_{\omega}, G_{i}\right)\right) \mid \mathcal{I}_{t}\right)$ - the integrity of past observations is continually discounted, new observations are fed in using minimum relative entropy inference - Eqn. 4. This yields a probability distribution over all possible outcomes that could occur if $X_{0}$ were to commit to a Delegate $(\cdot)$ proposal. $X_{0}$ then uses this distribution to decide whether or not to commit on the basis of the simple criterion: $\mathbf{P}\left(\operatorname{Acc}_{\mathrm{d}}\left(\left(X_{0}, \rho\right),\left(X_{\omega}, G_{i}\right)\right) \mid \mathcal{I}_{t}\right)>\alpha$ for some personal 'comfort factor' $\alpha$. The details of how this probability distribution is derived is the same as for $\operatorname{Agg}_{d}(\cdot)$ - this is described following.

\section{INTERACTION}

$X_{0}$ interacts with its collaborators $\left\{X_{i}\right\}_{i=1}^{n}$. It is assumed that goals are initially triggered externally to the system. For example, $X_{0}$ 's 'owner' may have an idea that she believes has value, and triggers an emergent process to explore the idea's worth. The interaction protocol is simple, if $X_{0}$ sends a Delegate $(\cdot)$ or a Join $(\cdot)$ message to $X_{\omega}$ then an interaction has commenced and continues until one agent sends an Accept(.) or a Quit(.) message. This assumes that agents respond in reasonable time which is fair in an essentially cooperative system.

To support the agreement-exchange process, $X_{0}$ has do two different things. First, it must respond to proposals received from $X_{\omega}$ - that is described in Sec. 5. Second, it must construct proposals, and possibly information, to send to $X_{\omega}$ - that is described now. Maximum entropy inference is used to 'fill in' missing values with the "maximally noncommittal" probability distribution. To illustrate this suppose that $X_{0}$ proposes to delegate a process to $X_{\omega}$. This process involves $X_{\omega}$ delivering - using an $\operatorname{Inform}(\cdot)$ message $-u$ chapters for a report in so-many days $v$. This section describes machinery for estimating the probabilities $\mathbf{P}\left(\operatorname{Agg}_{\mathrm{d}}\left(\left(X_{0}, u\right),\left(X_{\omega}, G_{v}\right)\right)\right.$ where the predicate:

- $\operatorname{Agg}_{\mathrm{d}}\left(\left(X_{0}, u\right),\left(X_{\omega}, G_{v}\right)\right)$ means " $X_{\omega}$ will accept $X_{0}$ 's delegation proposal $\left(\left(X_{0}, u\right),\left(X_{\omega}, G_{v}\right)\right)$ ".

$X_{0}$ assumes the following two preference relations for $X_{\omega}$, and $\mathcal{K}$ contains:

$$
\begin{aligned}
\kappa_{11}: \quad \forall x, y, z((x<y) \rightarrow \\
\left.\quad\left(\operatorname{Agg}_{\mathrm{d}}\left(\left(X_{0}, y\right),\left(X_{\omega}, G_{z}\right)\right) \rightarrow \operatorname{Agg}_{\mathrm{d}}\left(\left(X_{0}, x\right),\left(X_{\omega}, G_{z}\right)\right)\right)\right) \\
\kappa_{12}: \quad \forall x, y, z((x<y) \rightarrow \\
\left.\quad\left(\operatorname{Agg}_{\mathrm{d}}\left(\left(X_{0}, z\right),\left(X_{\omega}, G_{x}\right)\right) \rightarrow \operatorname{Agg}_{\mathrm{d}}\left(\left(X_{0}, z\right),\left(X_{\omega}, G_{y}\right)\right)\right)\right)
\end{aligned}
$$

As noted in Sec. 4.3, these sentences conveniently reduce the number of possible worlds. The two preference relations $\kappa_{11}$ and $\kappa_{12}$ induce a partial ordering on the sentence probabilities in the $\mathbf{P}\left(\operatorname{Agg}_{\mathrm{d}}\left(\left(X_{0}, u\right),\left(X_{\omega}, G_{v}\right)\right)\right)$ array. There are fifty-one possible worlds that are consistent with $\mathcal{K}$.

Suppose that $X_{0}$ has the following historical data on similar dealings with $X_{\omega}$. Three months ago $X_{\omega}$ asked for ten days to deliver four chapters. Two months ago $X_{0}$ proposed one day to deliver three chapters and $X_{\omega}$ refused. One month ago $X_{\omega}$ asked for eight days to deliver two chapters. $\mathcal{B}$ contains:

$$
\begin{aligned}
& \beta_{11}: \operatorname{Agg}_{\mathrm{d}}\left(\left(X_{0}, 4\right),\left(X_{\omega}, G_{10}\right)\right), \\
& \beta_{12}: \operatorname{Agg}_{\mathrm{d}}\left(\left(X_{0}, 3\right),\left(X_{\omega}, G_{1}\right)\right) \text { and } \\
& \beta_{13}: \operatorname{Agg}_{\mathrm{d}}\left(\left(X_{0}, 2\right),\left(X_{\omega}, G_{8}\right)\right),
\end{aligned}
$$

and assuming a $10 \%$ decay in integrity for each month: $\mathbf{P}\left(\beta_{11}\right)=0.7, \mathbf{P}\left(\beta_{12}\right)=0.2$ and $\mathbf{P}\left(\beta_{13}\right)=0.9$

Eqn. 3 is used to calculate the distribution $\mathbf{W}_{\{\mathcal{K}, \mathcal{B}\}}$ which shows that there are just five different probabilities in it. The probability matrix for the proposition:

$$
\operatorname{Agg}_{\mathrm{d}}\left(\left(X_{0}, u\right),\left(X_{\omega}, G_{v}\right)\right)
$$

is:

$\begin{array}{cccccc}v \backslash u & 1 & 2 & 3 & 4 & 5 \\ 11 & 0.9967 & 0.9607 & 0.8428 & 0.7066 & 0.3533 \\ 10 & 0.9803 & 0.9476 & 0.8330 & \mathbf{0 . 7 0 0 0} & 0.3500 \\ 9 & 0.9533 & 0.9238 & 0.8125 & 0.6828 & 0.3414 \\ 8 & 0.9262 & \mathbf{0 . 9 0 0 0} & 0.7920 & 0.6655 & 0.3328 \\ 7 & 0.8249 & 0.8019 & 0.7074 & 0.5945 & 0.2972 \\ 6 & 0.7235 & 0.7039 & 0.6228 & 0.5234 & 0.2617 \\ 5 & 0.6222 & 0.6058 & 0.5383 & 0.4523 & 0.2262 \\ 4 & 0.5208 & 0.5077 & 0.4537 & 0.3813 & 0.1906 \\ 3 & 0.4195 & 0.4096 & 0.3691 & 0.3102 & 0.1551 \\ 2 & 0.3181 & 0.3116 & 0.2846 & 0.2391 & 0.1196 \\ 1 & 0.2168 & 0.2135 & \mathbf{0 . 2 0 0 0} & 0.1681 & 0.0840\end{array}$


In this array, the derived sentence probabilities for the three sentences in $\mathcal{B}$ are shown in bold type; they are exactly their given values.

$X_{0}$ 's interaction strategy is a function $\mathbf{S}: \mathcal{K} \times \mathcal{B} \rightarrow \mathcal{A}$ where $\mathcal{A}$ is the set of actions that send Delegate $(\cdot)$, Join $(\cdot)$, $\operatorname{Accept}(\cdot), \operatorname{Reject}(\cdot), \operatorname{Inform}(\cdot)$ and Quit $(\cdot)$ messages to $X_{\omega}$. If $X_{0}$ sends any message to $X_{\omega}$ then she is giving $X_{\omega}$ information about herself.

\section{1 'Even-handed' collaboration}

An agent may be motivated to act for various reasons three are mentioned. First, if there are costs involved in the interaction due either to changes in the value of the interaction object with time or to the intrinsic cost of conducting the interaction itself. Second, if there is a risk of breakdown caused by a collaborator dropping out of a negotiation. Third, if the agent is concerned with establishing a sense of trust [13] with the collaborator - this could be the case in the establishment of a business relationship. Of these three reasons the last two are addressed here. The risk of breakdown may be reduced, and a sense of trust may be established, if the agent appears to its collaborator to be "approaching the interaction in an even-handed manner". One dimension of "appearing to be even-handed" is to be equitable with the value of information given to the collaborator. Various interaction strategies, both with and without breakdown, are described in [2], but they do not address this issue. An interaction strategy is described here that is founded on a principle of "equitable information gain". That is, $X_{0}$ attempts to respond to $X_{\omega}$ 's messages so that $X_{\omega}$ 's expected information gain similar to that which $X_{0}$ has received.

$X_{0}$ models $X_{\omega}$ by observing her actions, and inferring beliefs about her future actions in probability distributions such as $\mathbf{P}\left(\mathrm{Agg}_{\mathrm{d}}\right)$. $X_{0}$ measures the value of information that it receives from $X_{\omega}$ by the change in the entropy of this distribution as a result of representing that information in $\mathbf{P}\left(\mathrm{Agg}_{\mathrm{d}}\right) . \mathcal{I}_{t}$ is $X_{0}$ 's information base at time $t$. Suppose that $X_{0}$ then receives a message $\mu$ giving $\mathcal{I}_{t+1}$ at time $t+1$. Then the information in $\mu$ with respect to the information base $\mathcal{I}_{t}$ is:

$$
\mathbf{I}\left(\mu \mid \mathcal{I}_{t}\right)=\mathbf{H}\left(\operatorname{Agg}_{\mathrm{d}}\left(\mathcal{I}_{t}\right)\right)-\mathbf{H}\left(\operatorname{Agg}_{\mathrm{d}}\left(\mathcal{I}_{t+1}\right)\right)
$$

where the argument of $\operatorname{Agg}_{\mathrm{d}}(\cdot)$ denotes the state of the information base from which it was derived, and $\mathbf{H}\left(\operatorname{Agg}_{\mathrm{d}}(\cdot)\right)$ is the entropy of the underlying probability distribution from which $\mathrm{Agg}_{\mathrm{d}}$ is aggregated. An alternative way of measuring the value of information is as the Kullback-Leibler distance between the prior and the posterior distributions. If $\left(p_{i}\right)_{i=1}^{n}$ is the underlying probability distribution from which $\left.\operatorname{Agg}_{\mathrm{d}}\left(\mathcal{I}_{t}\right)\right)$ was aggregated and $\left(q_{i}\right)_{i=1}^{n}$ the distribution for $\left.\operatorname{Agg}_{\mathrm{d}}\left(\mathcal{I}_{t+1}\right)\right)$ then:

$$
\mathbf{I}\left(\mu \mid \mathcal{I}_{t}\right)=\sum_{i=1}^{n} p_{i} \log \frac{p_{i}}{q_{i}}
$$

More generally, $X_{0}$ measures the value of information received in a message, $\mu$, by the change in the entropy in its entire representation, $\mathcal{J}_{t}=\mathcal{K}_{t} \cup \mathcal{B}_{t}$, as a result of the receipt of that message; this is denoted by: $\Delta_{\mu}\left|\mathcal{J}_{t}^{\Pi}\right|$, where $\left|\mathcal{J}_{t}^{\Pi}\right|$ denotes the value (as negative entropy) of $X_{0}$ 's information in $\mathcal{J}$ at time $t$. Although both $X_{0}$ and $X_{\omega}$ will build their models of each other using the same data - the messages exchanged - the observed information gain will depend on the way in which each agent has represented this information. It is "not unreasonable to suggest" that these two representations should be similar. To support its attempts to achieve "equitable information gain" $X_{0}$ assumes that $X_{\omega}$ 's reasoning apparatus mirrors its own, and so is able to estimate the change in $X_{\omega}$ 's entropy as a result of sending a message $\mu$ to $X_{\omega}: \Delta_{\mu}\left|\mathcal{J}_{t}^{\Omega}\right|$. Suppose that $X_{0}$ receives a message $\mu=$ Delegate $(\cdot)$ from $X_{\omega}$ and observes an information gain of $\Delta_{\mu}\left|\mathcal{J}_{t}^{\Pi}\right|$. Suppose that $X_{0}$ wishes to reject this agreement by sending a counter-proposal, Delegate $(\cdot)$, that will give $X_{\omega}$ expected "equitable information gain". This is achieved by:

$$
\begin{aligned}
& \delta=\left\{\arg \max _{\delta}\right. \mathbf{P}\left(\operatorname{Acc}_{\mathrm{d}}(\delta) \mid \mathcal{I}_{t}\right) \geq \alpha \mid \\
&\left.\left(\Delta_{\text {Delegate }(\delta)}\left|\mathcal{J}_{t}^{\Omega}\right| \approx \Delta_{\mu}\left|\mathcal{J}_{t}^{\Pi}\right|\right)\right\}
\end{aligned}
$$

That is $X_{0}$ chooses the most acceptable agreement to herself that gives her collaborator expected "equitable information gain" provided that there is such an agreement. If there is not then $X_{0}$ chooses the best available compromise

$$
\delta=\left\{\arg \max _{\delta}\left(\Delta_{\text {Delegate }(\delta)}\left|\mathcal{J}_{t}^{\Omega}\right|\right) \mid \mathbf{P}\left(\operatorname{Acc}_{\mathrm{d}}(\delta) \mid \mathcal{I}_{t}\right) \geq \alpha\right\}
$$

provided there is such an agreement - this strategy is rather generous, it rates information gain ahead of personal acceptability. If there is not then $X_{0}$ quits.

\section{COLLABORATION}

The mechanism that $X_{0}$ uses for managing process delegation is described in full. Join $(\cdot)$ messages are managed similarly. This next section discusses the sorts of payoff measures and estimates that are available, and that are combined to give a value for the expected payoff vector $\underline{\nu}_{i}$ for each agent. Let $\mathbf{P}(A \gg)$ denote $A$ is the 'best choice' in terms of some combination of the parameter estimates described following. These measurements are then used by agent $X_{0}$ to determine $\mathbf{P}\left(X_{i} \gg\right)$, and then in turn to determine the delegation strategy $\left(p_{i}\right)_{i=1}^{n}$.

\subsection{The Performance Parameters}

Agent $X_{0}$ continually measures the performance of itself and of other agents in the system using four measures. Three are: time, cost and likelihood of success which are attached to all of its delegations-in and delegations-out. The last one is a value parameter that is attached to other agents. Time is the total time taken to termination. Cost is the actual cost of the of resources allocated. For example, the time that the agent — possibly with a human 'assistant' — actually spent working on that process. The likelihood of success is the probability that an agent will deliver its response within its constraints. The value parameter is the value added to a process by an agent. Unfortunately, value is often very difficult to measure - it is treated here by a subjective estimate delivered by users of the system.

The three parameters time, cost and likelihood of success are observed and recorded every time an agent, including $X_{0}$, delivers, or fails to deliver, its commitments. This generates a large amount of data whose significance can reasonably be expected to degrade over time. So a cumulative estimate only is retained. The integrity of information 'evaporates' as time goes by. If we have the set of observable outcomes as $O=\left\{o_{1}, o_{2}, \ldots, o_{m}\right\}$ then complete ignorance of the expected outcome means that our expectation over 
these outcomes is $\frac{1}{m}$ - i.e. the unconstrained maximum entropy distribution. This natural decay of information integrity is offset by new observations.

Given one of the parameters, $u$, with $m$ possible outcomes ${ }^{1}$, suppose that $\mathbf{P}^{t}\left(u^{\prime} \mid \delta\right)$ is the estimate at time $t$ of the probability that the actual outcome $u^{\prime}$ will be observed given that the agent being observed has committed to $\delta$. Suppose that $X_{0}$ observes the actual outcome $r$, on the basis of this outcome $X_{0}$ believes that the probability of $r$ being observed at the next time is $g_{r}$. Then let $\mathbf{P}_{g_{r}}^{t}\left(u^{\prime} \mid \delta\right)$ be the posterior minimum relative entropy distribution calculated using Eqn. 4 with prior distribution $\mathbf{P}^{t}\left(u^{\prime} \mid \delta\right)$ and satisfying the constraint that $\mathbf{P}_{g_{r}}^{t}(r \mid \delta)=g_{r}$. Then update $\mathbf{P}^{t}\left(u^{\prime} \mid \delta\right)$ with:

$$
\mathbf{P}^{t+1}\left(u^{\prime} \mid \delta\right)=\frac{1-\rho}{n}+\rho \cdot \mathbf{P}_{g_{r}}^{t}\left(u^{\prime} \mid \delta\right)
$$

This equation determines the development of $\mathbf{P}^{t}\left(u^{\prime} \mid \delta\right)$ for some large $\rho \in[0,1]$.

$X_{0}$ uses the method in Eqn. 5 to update its estimates for all probability distributions representing each of the agents that it deals with. For example, if $\mathbf{P}^{t}(\cdot)$ is $X_{0}$ 's estimate of the time that $X_{\omega}$ will take to deliver on a particular type of agreement. Suppose that at time $t, X_{\omega}$ delivers her response after having taken time $u$. Then $X_{0}$ attaches a belief (i.e. a sentence probability) to the proposition that this is how $X_{\omega}$ will behave at time $t+1$. This becomes the constraint in the minimum relative entropy calculation and then Eqn. 5 gives $\mathbf{P}^{t+1}(\cdot)$.

The process delegation problem belongs to the class of resource allocation games which are inspired by the 'El Farol Bar' problem — see [6] for recent work.

\subsection{Choosing the 'best' collaborator}

The probability distributions described above may be used to determine the probability that one agent is a better choice than another by calculating the probability that one random variable is greater than another in the usual way. This method may be extended to estimate the probability that one agent is a better choice than a number of other agents. For example, if there are three agents to choose from, $A, B$, and $C$, then:

$$
\begin{aligned}
\mathbf{P}(A \gg) & =\mathbf{P}((A \gg B) \wedge(A \gg C)) \\
& =\mathbf{P}(A \gg B) \times \mathbf{P}((A \gg C) \mid(A \gg B))
\end{aligned}
$$

The difficulty with this expression is that there is no direct way of estimating the second, conditional probability. This expression shows that:

$$
\mathbf{P}(A \gg B) \times \mathbf{P}(A \gg C) \leq \mathbf{P}(A \gg) \leq \mathbf{P}(A \gg B)
$$

By considering the same expression with $B$ and $C$ interchanged:

$$
\mathbf{P}(A \gg B) \times \mathbf{P}(A \gg C) \leq \mathbf{P}(A \gg) \leq \mathbf{P}(A \gg C)
$$

and so:

$$
\mathbf{P}(A \gg) \leq \min [\mathbf{P}(A \gg B), \mathbf{P}(A \gg C)]
$$

\footnotetext{
${ }^{1}$ The success parameter has only two possible outcomes 'succeed' and 'fail'.
}

So for some $\tau_{A} \in[0,1]$ :

$$
\begin{gathered}
\mathbf{P}(A \gg)=\mathbf{P}(A \gg B) \times \mathbf{P}(A \gg C)+ \\
\tau_{A} \times[\min [\mathbf{P}(A \gg B), \mathbf{P}(A \gg C)]- \\
\quad \mathbf{P}(A \gg B) \times \mathbf{P}(A \gg C)]
\end{gathered}
$$

Similar expressions may be constructed for the probabilities that $B$ and $C$ are the best agents respectively. This is as far as probability theory can go without making some assumptions. To proceed assume that: $\tau_{A}=\tau_{B}=\tau_{C}=\tau$; this assumption is unlikely to be valid, but it should not be "too far" from correct. Either $A$ or $B$ or $C$ will be the best plan, so the sum of the three expressions for the probabilities of $A, B$ and $C$ being the "best" plan will be unity. Hence:

$\tau=\frac{1-d}{q-d}$ where:

$$
\begin{aligned}
d= & {[(\mathbf{P}(A \gg B) \times \mathbf{P}(A \gg C))+} \\
& (\mathbf{P}(B \gg C) \times \mathbf{P}(B \gg A))+(\mathbf{P}(C \gg A) \times \mathbf{P}(C \gg B))] \\
q= & {[\min [\mathbf{P}(A \gg B), \mathbf{P}(A \gg C)]+} \\
& \min [\mathbf{P}(B \gg C), \mathbf{P}(B \gg A)]+ \\
& \min [\mathbf{P}(C \gg A), \mathbf{P}(C \gg B)]]
\end{aligned}
$$

This expression for $\tau$ is messy but is easy to calculate. The probability that each of the three agents $A, B$ and $C$ is the "best" choice is $\mathbf{P}(A \gg), \mathbf{P}(B \gg)$ and $\mathbf{P}(C \gg)$.

An alternative to the above is simply to use Eqn. 2 to estimate the probability of the propositions that each of the agents is the 'best' collaborator. That is, for agent $A$ :

$$
\mathbf{P}(A \gg)=\mathbf{P}_{\mathcal{W}_{\{\mathcal{K}, \mathcal{B}\}}}(A \gg)
$$

To calculate this probability then requires the calculation of the maximum entropy distribution that is consistent with $\mathcal{K}$ and $\mathcal{B}$, and then simply adding up the probabilities in that distribution that are associated with possible worlds in which agent $A$ is the 'best'. This alternative approach involves a maximum entropy calculation whereas the above approach does not.

\subsection{Delegation Strategy}

A delegation strategy is a probability distribution $\left\{p_{i}\right\}_{i=1}^{n}$ that determines who from $\left\{X_{i}\right\}_{i=1}^{n}$ to offer responsibility to for doing what. The delegation strategy determines "who does what" stochastically by determining the $\left\{p_{i}\right\}_{i=1}^{n}$ where $p_{i}$ is the probability that the i'th agent will be selected. The choice of the agent to delegate to is then made with these probabilities. The expression of the delegation strategy in terms of probabilities enables the strategy to balance conflicting goals, such as achieving process quality and process efficiency.

A greedy strategy best picks the agent that promises greatest returns:

$$
p_{i}= \begin{cases}\frac{1}{m} & \text { if } X_{i} \text { is such that } \mathbf{P}\left(X_{i} \gg\right) \text { is maximal } \\ 0 & \text { otherwise }\end{cases}
$$

where $m$ is such that there are $m$ agents for whom $\mathbf{P}\left(X_{i} \gg\right)$ is maximal. This strategy attempts to maximise expected payoff but it is short-sighted in that it rewards success with work - although it is not uncommon in practice. Another strategy prob also favours high payoff, but gives all agents a chance to prove themselves, sooner or later, and is defined by: $p_{i}=\mathbf{P}\left(X_{i} \gg\right)$. The strategy random is equitable, and picks agents by: $p_{i}=\frac{1}{n}$. 
An admissible delegation strategy has the properties:

$$
\begin{aligned}
& \text { if } \mathbf{P}\left(X_{i} \gg\right)>\mathbf{P}\left(X_{j} \gg\right) \text { then } p_{i}>p_{j} \\
& \text { if } \mathbf{P}\left(X_{i} \gg\right)=\mathbf{P}\left(X_{j} \gg\right) \text { then } p_{i}=p_{j} \\
& (\forall i) p_{i}>0 \text { and } \sum_{i} p_{i}=1
\end{aligned}
$$

So best and random are not admissible strategies but prob is admissible.

$\mathbf{P}\left(X_{i} \gg\right)$ is the probability that $X_{i}$ is the 'best' choice. The strategy best that continually choses the 'best' on the basis of historic data is flawed because an agent who "goes through a bad patch" may never be chosen - this means that if an agent wants "the quiet life" then all it would have to do is to make a series of mistakes. The delegation strategy prob is a compromise between being equitable and utility optimisation - it chooses agents with probability $p_{i}=\mathbf{P}\left(X_{i} \gg\right)$. That is, the probability that $X_{0}$ will attempt to delegate a process to $X_{\omega}$ is equal to the probability that $X_{0}$ estimates $X_{\omega}$ to be the 'best' choice for the job.

\section{CONCLUSION}

Emergent processes are collaborative business processes whose execution is determined by the prior knowledge of the agents involved and by the knowledge that emerges during a process instance. In an emergent process, the process goal may mutate, and so does not provide clear direction for process management. As emergent processes may involve informal interaction, there is a limit to the extent to which the processes per se can be "managed". However, the collaboration can be managed. The solution proposed builds on ideas from information theory and entropy-based inference. These inference methods are logic-based and so operate with multi-issue interaction with ease - this is particularly significant for the interactions involved in these high-level processes. The establishment of a sense of trust contributes to the establishment of business relationships and to preventing breakdown during interaction. This is addressed by the agents attempting to exhibit 'fair play' by applying the principle of equitable information revelation.

To manage collaboration the agent is equipped with: Delegate $(\cdot), \operatorname{Join}(\cdot), \operatorname{Accept}(\cdot), \operatorname{Reject}(\cdot), \operatorname{Inform}(\cdot)$ and Quit(.) interaction predicates. This discussion has focussed on the Delegate $(\cdot)$ predicate. Join $(\cdot)$ is dealt with similarly. $\operatorname{Inform}(\cdot)$ is used to satisfy a delegation goal, and the remaining predicates are necessary to support the interaction.

The agents in the system are 'essentially benevolent' they do no necessarily require motivation to contribute to a collaborative group or to take responsibility for a subprocess. Despite this, the agents also have a responsibility to their own user. So our agent does not attempt to secondguess the motives of the other agents in the system. Instead it takes advantage of the large amount of readily available information concerning past performance to estimate, using maximum entropy methods, expectations about future performance. The information in the system is based on past observations and so its integrity is in a permanent state of decay [1]. The agent selects its collaborators from the system by using a stochastic strategy. This strategy identifies a collaborator with a probability that is equal to the agent's estimate that she is the 'best' choice. This strategy provides a reasonable balance between getting things done in the best way and spreading the work around.

\section{REFERENCES}

[1] D. Bernhardt and J. Miao. Informed trading when information becomes stale. The Journal of Finance, LIX(1), February 2004.

[2] J. Debenham. Bargaining with information. In N. Jennings, C. Sierra, L. Sonenberg, and M. Tambe, editors, Proceedings Third International Conference on Autonomous Agents and Multi Agent Systems AAMAS-2004, pages 664 - 671. ACM, July 2004.

[3] P. Dourish. Using metalevel techniques in a flexible toolkit for CSCW applications. ACM Transactions on Computer-Human Interaction (TOCHI), 5(2):109 155, June 1998.

[4] M. Esteva, J. Padget, and C. Sierra. Formalizing a languages for institutions and norms. In J. Meyer and M. Tambe, editors, Intelligent Agents VIII, pages 348 - 366. Springer-Verlag, Berlin, Germany, 2002.

[5] L. Fischer. The Workflow Handbook 2003. Future Strategies Inc., 2003.

[6] A. Galstyan, S. Kolar, and K. Lerman. Resource allocation games with changing resource capacities. In Proceedings of the second international joint conference on Autonomous agents and multiagent systems AAMAS-03, pages 145 - 152, 2003.

[7] J. Halpern. Reasoning about Uncertainty. MIT Press, 2003.

[8] M. Huhns and M. Singh. Managing heterogeneous transaction workflows with cooperating agents. In N. Jennings and M. Wooldridge, editors, Agent Technology: Foundations, Applications and Markets, pages 219 - 239. Springer-Verlag: Berlin, Germany, 1998.

[9] A. Jain, M. Aparicio, and M. Singh. Agents for process coherence in virtual enterprises. Communications of the ACM, 42(3):62 - 69, 1999.

[10] E. Jaynes. Probability Theory - The Logic of Science. Cambridge University Press, 2003.

[11] N. Jennings, P. Faratin, T. Norman, P. O'Brien, and B. Odgers. Autonomous agents for business process management. Int. Journal of Applied Artificial Intelligence, 142(2):145 - 189, 2000.

[12] D. MacKay. Information Theory, Inference and Learning Algorithms. Cambridge University Press, 2003.

[13] S. Ramchurn, N. Jennings, C. Sierra, and L. Godo. A computational trust model for multi-agent interactions based on confidence and reputation. In Proceedings 5th Int. Workshop on Deception, Fraud and Trust in Agent Societies, 2003.

[14] M. Singh. Business Process Management: A Killer Ap for Agents? In N. Jennings, C. Sierra, L. Sonenberg, and M. Tambe, editors, Proceedings Third International Conference on Autonomous Agents and Multi Agent Systems AAMAS-2004, pages 26-27. ACM, July 2004.

[15] H. Smith and P. Fingar. Business Process Management (BPM): The Third Wave. Meghan-Kiffer Press, 2003.

[16] W. van der Aalst and K. van Hee. Workflow Management: Models, Methods, and Systems. The MIT Press, 2002.

[17] M. Wooldridge. Multiagent Systems. Wiley, 2002. 